\title{
Ultrathin antiferromagnetic films on a ferromagnetic substrate: a first-principles study of $\mathrm{Mn}$ on $\mathrm{Fe}(001)$
}

\author{
A Ernst ${ }^{1}, \mathrm{~J} \mathrm{Henk}^{1}$ and $\mathrm{R} \mathrm{K}$ Thapa ${ }^{2}$ \\ ${ }^{1}$ Max-Planck-Institut für Mikrostrukturphysik, Weinberg 2, D-06120 Halle (Saale), Germany \\ 2 Condensed Matter Theory Research Group, Physics Department, Pachhunga University \\ College, Mizoram University, Aizawl 796001 Mizoram, India \\ E-mail: henk@mpi-halle.de
}

Received 8 April 2005

Published 13 May 2005

Online at stacks.iop.org/JPhysCM/17/3269

\begin{abstract}
The electronic and magnetic structures of Mn films with thicknesses of up to 12 monolayers on $\mathrm{Fe}(001)$ are investigated by means of first-principles calculations, with a focus on the thicker films. The Mn films show an in-layer antiferromagnetic structure and couple antiferromagnetically to the $\mathrm{Fe}$ substrate. The magnetic moments at the Mn surface and at the $\mathrm{Mn}-\mathrm{Fe}$ interface are significantly enhanced, as compared to the respective bulk values. Several surface states are found at the centre of the two-dimensional Brillouin zone, some of which might explain recent experimental scanning tunnelling spectroscopy results.
\end{abstract}

(Some figures in this article are in colour only in the electronic version)

\section{Introduction}

The enormous progress in preparation techniques for ultrathin films has a tremendous impact on the development of surface physics and of practical applications [1]. Especially, magnetic multilayers led to new electronic devices which, for instance, are based on giant magnetoresistance and on tunnel magneto-resistance $[2,3]$. Therefore, properties of ultrathin magnetic films are of particular importance, both from the experimental and from the theoretical point of view. Compared to bulk systems, these systems show novel magnetic and electronic features due to the reduced dimension. In this respect, $\mathrm{Mn}$ films on $\mathrm{Fe}(001)$ are worth mentioning because they reveal a delicate interplay of the geometric, the electronic, and the magnetic structure.

The magnetic properties of ultrathin $\mathrm{Mn}$ films on $\mathrm{Fe}(001)$ have been controversially discussed in the literature. Being an antiferromagnet in the bulk itself, a question arose of how the magnetic configuration of $\mathrm{Mn}$ is changed upon attaching to a ferromagnetic $\mathrm{Fe}$ substrate [4]. Another issue involved the magnetic coupling of the Mn films to the Fe substrate. 
Theoretical investigations of mono- and bilayers showed both ferromagnetic (FM) [5] and antiferromagnetic (AFM) coupling [6] (cf also [7, 8]). Further, Wu and Freeman reported that the magnetic ground state of a Mn monolayer is given by a $\mathrm{c}(2 \times 2)$ reconstruction in which there is AFM order within the Mn monolayer [9]. This finding was in general supported by Asada et al but a $\mathrm{p}(2 \times 2)$ reconstruction with FM order within the Mn film showed a slightly lower total energy [10]. A c $(2 \times 2)$ ground state was also found by Zenia et al, which upon oxygen coverage is turned into an AFM $\mathrm{p}(1 \times 1)$ structure [11]. Considering bulk and multilayer systems, Stoeffler and Gautier reported on both FM and AFM configurations for the Fe-Mn interface [12], the former being energetically more stable. Purcell et al found an AFM ground state for both hypothetical bulk body-centred tetragonal Mn and Fe-Mn multilayers in calculations for the experimental lattice spacings [13]. Evidently, the magnetic properties depend significantly on the lattice spacings, as was supported by $\mathrm{Wu}$ and Freeman [6,9] (cf also $[14,15])$. In summary, the magnetic structures of Mn mono- and bilayers on Fe(001) are complicated and are still under consideration (a review of electronic-structure calculations for thin films, also for $\mathrm{Mn} / \mathrm{Fe}(001)$, is given by Vega et al [16]).

As inconsistencies showed up among theoretical investigations, they were present among various experiments, too. For example, x-ray magnetic circular dichroism and He-ion scattering experiments concluded on both FM and AFM coupling of low-coverage Mn films to the Fe substrate [17-20]. For thicker films, there were indications of an AFM configuration within the topmost Mn layer [19, 20]. However, spin-polarized electron-energyloss spectroscopy and SEMPA (scanning electron microscopy with polarization analysis) experiments on a $\mathrm{Mn}$ wedge grown on $\mathrm{Fe}(001)$ provided evidence that the covering films display an in-layer AFM (L-AFM) structure [21, 22]. This finding was further corroborated by scanning tunnelling microscopy (STM) [23]: changes in step heights start in films $4 \mathrm{ML}$ (monolayer) thick, their period of two layers possibly being related to an L-AFM configuration. A structural change at 3 ML thickness was also observed by Andrieu et al $[17,24]$, reporting in addition on strong sensitivity of the magnetic properties on growth conditions [25].

Summarizing at this point, the magnetic configuration within the $\mathrm{Mn}$ films and their coupling to the $\mathrm{Fe}(001)$ substrate was not clearly resolved, either theoretically or experimentally [26]. The sensitivity to parameters in theory-like lattice spacings-is accompanied by that to preparation conditions in experiment [21, 27]. However, for thicker films a trend towards an L-AFM structure was established [21, 28, 29].

Up to now, first-principles calculations for $\mathrm{Mn} / \mathrm{Fe}(001)$ were performed mainly for low coverages, especially for monolayers and bilayers. In a recent publication, Martínez et al reported on ab initio calculations for Mn films 6 and 7 ML thick [30]. Several collinear magnetic structures were found which reveal a competition between FM and L-AFM ordering. As in that investigation, the focus of the present theoretical work lies on thicker films, in which the L-AFM structure has been clearly observed in experiment. An unresolved issue still concerns the coupling to the Fe substrate. Further, a question arises whether the electronic and magnetic structures can be viewed as being composed of an interface part, a Mn bulk-like part, and a surface part.

For Mn films with L-AFM structure, Yamada et al reported on a maximum at $+0.8 \mathrm{eV}$ in $\mathrm{d} I / \mathrm{d} V$ spectra of scanning tunnelling spectroscopy (STS) experiments $[31,32]$. This feature was explained by 'minority' surface states at $0.27 \mathrm{eV}$ and at $0.50 \mathrm{eV}$ relative to the Fermi energy (the term 'minority' refers here to the magnetization direction of the outermost Mn layer). Although it was energetically closer, a third surface state at $0.87 \mathrm{eV}$ was considered not to contribute to the observed maximum. These surface states were computed ab initio for an eight-layer slab of L-AFM Mn layers [33]. They show up at the centre of the two-dimensional Brillouin zone and possess $\mathrm{d}_{z^{2}}$ character, with a charge distribution projected into the vacuum. 
We note in passing that Bischoff reported on a surface state at $+0.35 \mathrm{eV}$ in STS experiments on $\mathrm{p}(1 \times 1) \mathrm{Mn}$ islands [23].

The difference in energies between the theoretical surface-state energies and the maximum in the STS experiment seems to be unsatisfactorily large. Therefore, there appears to be a need for a first-principles investigation which takes into account both the Fe substrate and the comparably thick Mn film. The present study reports on such calculations.

The paper is organized as follows. Details of the numerical approach are presented in section 2. The results are discussed in section 3, focusing first on charge profiles (section 3.1) and magnetic profiles (section 3.2). More details, in particular on surface states, are provided by the density of states (section 3.3) as well as by spectral densities (section 3.4).

\section{Computational details}

The electronic structures of Mn films on $\mathrm{Fe}(001)$ were computed from first principles using the local spin-density approximation of density-functional theory with Perdew-Wang exchangecorrelation potential [34]. The scalar-relativistic Korringa-Kohn-Rostoker (KKR) method was applied to semi-infinite systems, hence taking into account the appropriate boundary conditions (see [35] for details).

In a first step, the muffin-tin potential of bulk Fe was computed. Having thus fixed this potential and the Fermi energy, the self-consistent procedure was applied to the semi-infinite film-substrate systems. Here, the potentials of three vacuum layers, the Mn layers of the film, and the six adjacent Fe substrate layers were computed self-consistently. The number of Fe substrate layers of which the potentials were allowed to differ from the Fe-bulk potential was determined by considering the convergence of the charge and magnetic profiles (see sections 3.1 and 3.2 below) upon increasing this number. The same procedure applied for the vacuum region. The Mn layers were assumed to continue the bcc structure of Fe (with lattice constant 5.41 Bohr radii) but with layer-dependent interlayer distances. The parameters of the resulting body-centred tetragonal (bct) structure were taken from experimental results reported in [31]. Intermixing at the $\mathrm{Mn}-\mathrm{Fe}$ interface was not taken into account, in agreement with low-temperature growth conditions [23]. The thickness of the Mn films was between $n=1$ and 12 atomic layers, in order to be in the range present in experiments, expressly that of [32]. For comparison, the electronic structure of the Fe(001) surface was computed self-consistently, too $(n=0)$.

The $a b$ initio calculations provide details of the electronic structure by means of the spin-, layer- and wavevector-resolved Bloch spectral density

$$
N_{l}^{\tau}\left(E, \boldsymbol{k}_{\|}\right)=-\frac{1}{\pi} \operatorname{Im} \operatorname{Tr} G_{\tau \tau}^{+}\left(E, \boldsymbol{k}_{\|} ; \boldsymbol{r}-\boldsymbol{R}_{l}, \boldsymbol{r}-\boldsymbol{R}_{l}\right), \quad \tau=\uparrow, \downarrow,
$$

where the trace $\operatorname{Tr}$ stands for integration over a muffin-tin sphere located at $\boldsymbol{R}_{l}$ in layer $l$. $G_{\tau \tau}^{+}\left(E, \boldsymbol{k}_{\|} ; \boldsymbol{r}-\boldsymbol{R}_{l}, \boldsymbol{r}^{\prime}-\boldsymbol{R}_{l}\right)$ is the + side-limit of the layer-diagonal Green function of electrons with spin orientation $\tau$ at energy $E$ and in-plane wavevector $\boldsymbol{k}_{\|}=\left(k_{x}, k_{y}\right)$ [36]. In all cases discussed in the following, the label $\uparrow$ ('spin-up') refers to the magnetic-moment orientation of the Fe majority electrons, whereas $\downarrow$ ('spin-down') refers to that of the minority electrons. The outermost Fe layer (adjacent to the Mn film) has in all cases the index $l=0$. Going towards the bulk, $l$ decreases. Hence, the surface layer of an $n$-layer Mn film is indexed by $l=n$. Further insight is gained by decomposing $N_{l}^{\tau}\left(E, \boldsymbol{k}_{\|}\right)$with respect to angular momentum or point-group representation.

The density of states $D_{l}^{\tau}(E)$ is obtained by integrating $N_{l}^{\tau}\left(E, \boldsymbol{k}_{\|}\right)$over the two-dimensional Brillouin zone (2BZ),

$$
D_{l}^{\tau}(E)=\int_{2 \mathrm{BZ}} N_{l}^{\tau}\left(E, \boldsymbol{k}_{\|}\right) \mathrm{d} k_{\|}^{2}, \quad \tau=\uparrow, \downarrow .
$$


Particularly valuable tools for the analysis of the electronic and magnetic structure are charge and magnetic profiles $\left\{\rho_{l}\right\}$ and $\left\{m_{l}\right\}$ [37]:

$$
\begin{aligned}
& \rho_{l}=\int_{-\infty}^{E_{\mathrm{F}}}\left[D_{l}^{\uparrow}(E)+D_{l}^{\downarrow}(E)\right] \mathrm{d} E, \\
& m_{l}=\int_{-\infty}^{E_{\mathrm{F}}}\left[D_{l}^{\uparrow}(E)-D_{l}^{\downarrow}(E)\right] \mathrm{d} E .
\end{aligned}
$$

In view of identifying surface states, it turned out to be helpful to 'resect' potentials from the 12 ML Mn film and to compute from these auxiliary electronic structures. Surface states are located at the vacuum-film boundary and, hence, have to be energetically positioned in a bandgap of the point-group representation they belong to (for example, the 'minority' $\mathrm{d}_{z^{2}}$ surface states reported in [32] belong to the $\Delta_{1}^{\downarrow}$ representation). To obtain these bandgaps, the potentials of the central layers of the $12 \mathrm{ML}$ Mn film were cut out in order to form a hypothetical L-AFM bulk. This 'cut and paste' procedure was justified a posteriori by successfully observing the bandgaps of the bct-Mn bulk also in the ab initio results for the thicker Mn films. As a second criterion, the spectral weight-as given by the Bloch spectral densities $N_{l}^{\tau}\left(E, \boldsymbol{k}_{\|}\right)$- has to decay rapidly in a direction away from the surface layer.

In order to check whether our KKR approach can reproduce earlier results on $\mathrm{Mn}$, the magnetic structure of bulk bct $\mathrm{Mn}$ (with $c / a$ ratio 1.14 ) was calculated. The ground-state configuration was obtained from the total energy, which clearly prefers the antiferromagnetic $\mathrm{c}(2 \times 2)$ phase. The antiferromagnetic $\mathrm{p}(2 \times 1)$, the ferromagnetic, and the paramagnetic phases show larger total energies, in agreement with earlier work [15].

\section{Results and discussion}

Before turning to the results for Mn films on $\mathrm{Fe}(001)$, a few comments on the self-consistent calculations are in order. For the 1 and $2 \mathrm{ML}$ films, the calculations always converged to the L-AFM phase, with Mn layer 1 coupling AFM to the Fe substrate. (Focusing on thicker Mn films, note that these and the following results were obtained for $(1 \times 1)$ unit cells [30]. Hence, the calculations reported in the present paper are not supposed to reproduce the $(2 \times 2)$ ground-state reconstructions observed in other investigations for Mn monolayers [9-11].) For thicker films, we found also other magnetic phases which are worth investigating in a future publication (see also [30]). However, the AFM coupling of the Mn layers adjacent to the Fe substrate was observed in all our self-consistent calculations, even when starting from a completely FM configuration. In this paper, we focus on the L-AFM phase, as established by experiments and other theoretical work (see section 1 for references).

\subsection{Charge profiles}

First evidence of whether the electronic and magnetic structure of $\mathrm{Mn} / \mathrm{Fe}(001)$ can be decomposed into an interface part, a Mn-bulk part, and a surface part is provided by inspection of the charge profiles $\left\{\rho_{l}\right\}$, as defined in (3). From figure 1 it is obvious that the shape of the profiles does not change considerably for films thicker than about $6 \mathrm{ML}$. For these films, the Mn-bulk part comprises the 'flat' region between the interface part and the surface part. It displays oscillations with comparably small amplitude and a wavelength of $2 \mathrm{ML}$. The interface part extends from layer -2 (Fe) to layer $+3(\mathrm{Mn})$ and comprises a charge increase at Fe layer -1 (less than 0.1 electrons). Within the Mn films, a decrease at layer 1 (about -0.13 electrons) and an increase at layer 3 (about 0.11 electrons) is observed. The surface part consists mainly of the maximum at the outermost Mn layer (about 0.5 electrons) and is 


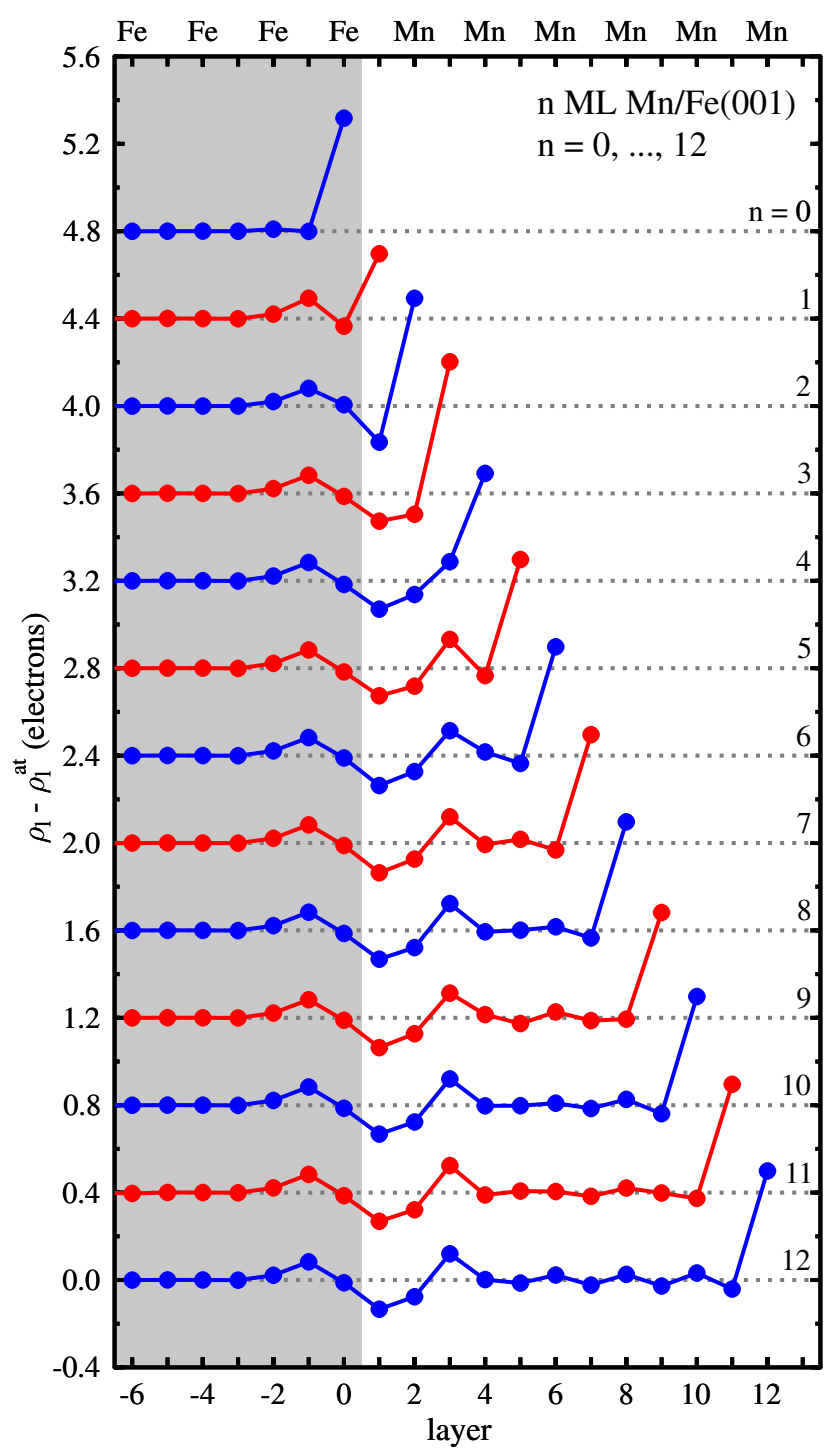

Figure 1. Charge profiles $\left\{\rho_{l}\right\}$ of $\mathrm{Mn}$ films on Fe(001) with thicknesses $n$ from 0 ML (top) to $12 \mathrm{ML}$ (bottom), as indicated on the right. The charge $\rho_{l}$ integrated over the muffin-tin spheres of each layer with index $l$ (indicated at the bottom and on the top) is displayed as difference to the nominal atomic charge $\rho_{l}^{\text {at }}\left(\rho_{l}^{\text {at }}=25\right.$ electrons and $\rho_{l}^{\text {at }}=26$ electrons for Mn and Fe, respectively $)$. The offset between adjacent profiles is 0.4 electrons. The range of the Fe substrate is marked grey, charges in the vacuum layers are not depicted.

already present for the very thin films. For films thinner than $6 \mathrm{ML}$ a Mn-bulk part cannot be established since the interface and the surface parts overlap.

With regard to the magnetic configurations, it appears interesting to decompose the charge profiles with respect to angular momentum and spin. In both Fe and $\mathrm{Mn}$, the d electrons carry the magnetic moment. The s and p electrons can be viewed as being polarized by the former. By this examination it is made possible to relate the magnetic profiles (discussed in section 3.2) and the charge redistribution at the $\mathrm{Mn}-\mathrm{Fe}$ interface to electrons with specific quantum numbers. 


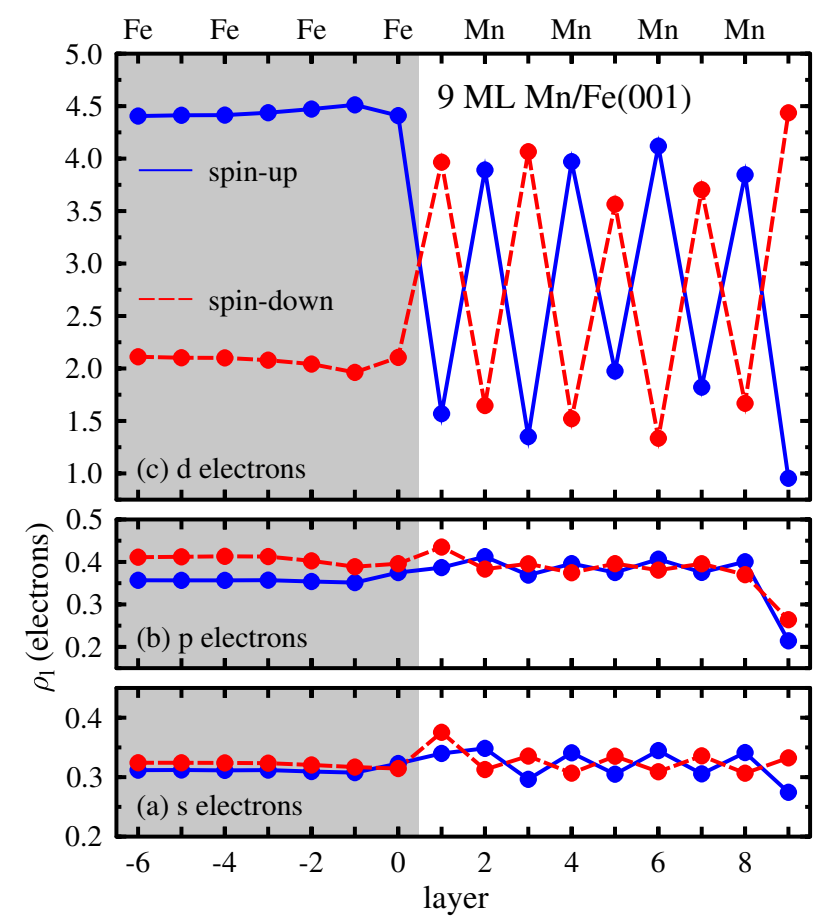

Figure 2. Spin- and angular-momentum-resolved charge profile $\left\{\rho_{l}\right\}$ of a 9 ML Mn film on Fe(001). The valence charge for s ((a), bottom), p ((b), centre), and d electrons ((c), top) is decomposed into up (solid) and down (dashed) spin orientation. The atomic species associated with each layer $l$ is indicated at the top; the region of the Fe substrate is marked grey.

As an example, the profiles for the $9 \mathrm{ML}$ film are presented in figure 2. As is evident from panel (c), the magnetism is carried mainly by the delectrons of Fe and $\mathrm{Mn}$. The larger number of spin-up electrons (blue) compared to that of spin-down electrons (red) in the substrate indicates the ferromagnetic ordering of Fe. In the Mn film, however, an alternating sequence is observed, with a surplus of spin-down (spin-up) electrons in odd-index (even-index) layers. This charge profile indicates an L-AFM configuration, with an AFM coupling to the Fe substrate.

The $\mathrm{s}$ and $\mathrm{p}$ electrons in the Fe substrate show the opposite spin orientation with respect to that of the d electrons, i.e., there is a surplus of spin-down $\mathrm{s}$ and $\mathrm{p}$ electrons. This reversal is, however, not found in the Mn film. In order to explain this finding, the electronic structures of the FM and L-AFM bulk configurations were computed for both Fe and Mn. For both elements, the band width in the L-AFM phase is reduced with respect to that of the FM case ('band narrowing'; cf [38]). Further, a pseudo-gap at about $-0.8 \mathrm{eV}$ is formed, accompanied by a shift of spectral weight towards above the Fermi energy. This rearrangement of spectral weight in conjunction with the reduced exchange splitting yields that the $\mathrm{s}$ and $\mathrm{p}$ electrons show the same spin orientation as the d electrons. Hence, the different spin orientations of $\mathrm{s}$ and $\mathrm{p}$ electrons with respect to the d electrons in the Fe substrate and in the Mn film are a manifestation of the different magnetic arrangements (FM versus L-AFM). We note in passing that the strong tendency of Mn towards antiferromagnetism shows up in the magnetic moments. For $\mathrm{Mn}$, it is less in the FM configuration than in the L-AFM configuration $\left(1.02 \mu_{\mathrm{B}}\right.$ versus $\left.2.31 \mu_{\mathrm{B}}\right)$, whereas for Fe the opposite is true $\left(2.24 \mu_{\mathrm{B}}\right.$ versus $\left.1.69 \mu_{\mathrm{B}}\right)$. 


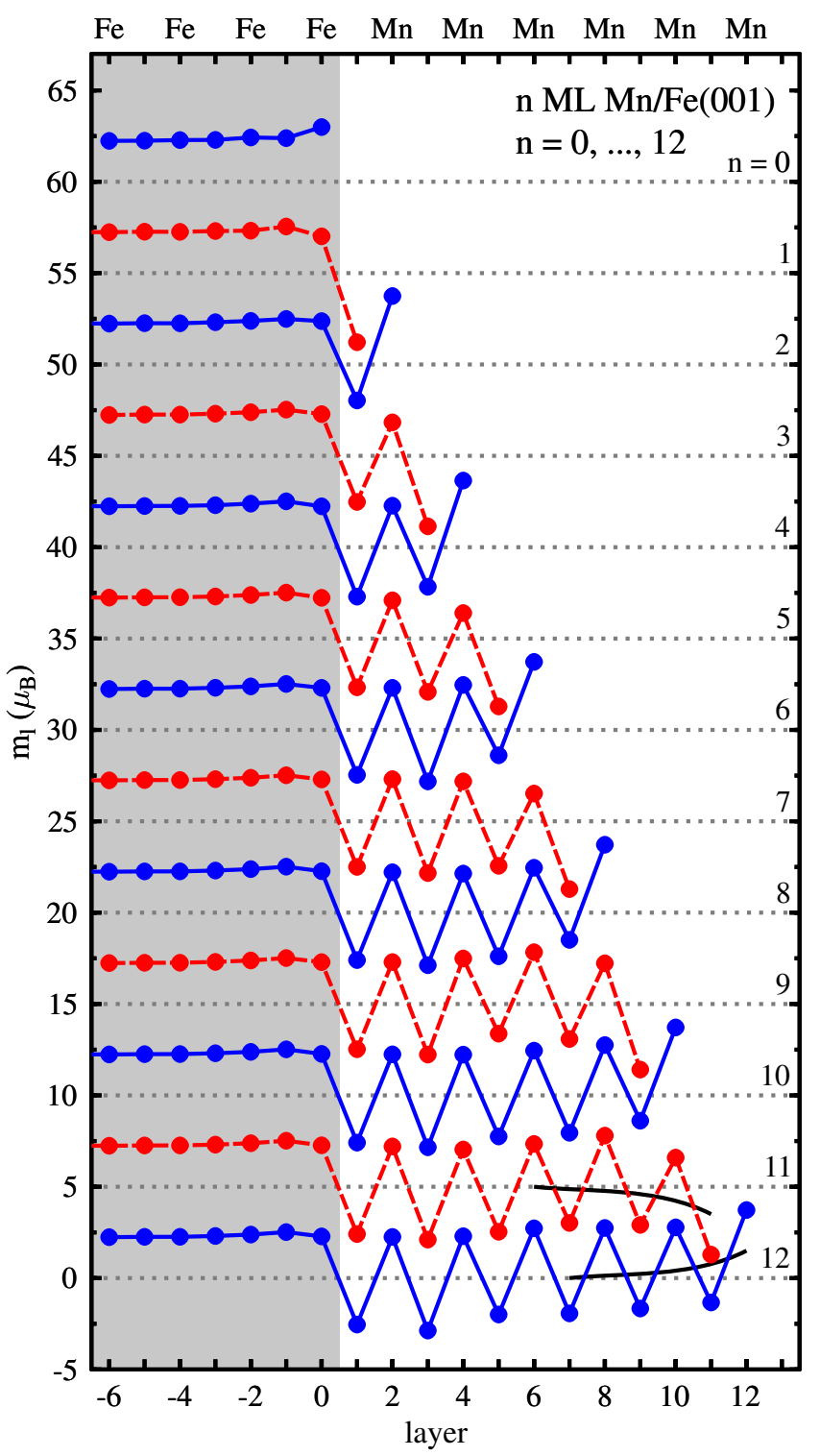

Figure 3. Magnetic profiles $\left\{m_{l}\right\}$ of Mn films on $\mathrm{Fe}(001)$ with thicknesses $n$ from $0 \mathrm{ML}$ (top) to $12 \mathrm{ML}$ (bottom), as indicated on the right. The magnetic moment $m_{l}$ integrated over the muffin-tin spheres of each layer with index $l$ (indicated at the bottom and at the top) is displayed, alternating solid and dashed for even and odd numbers of covering Mn layers. The offset between adjacent profiles is $5 \mu_{\mathrm{B}}$. The range of the Fe substrate is marked grey. Short curved lines at the $11 \mathrm{ML}$ and the 12 ML films visualize schematically the 'bending' of the magnetic profiles.

\subsection{Magnetic profiles}

Now, we turn to the discussion of the magnetic profiles $\left\{m_{l}\right\}$, as defined in (4). A first glance at figure 3 establishes immediately the L-AFM configurations in the Mn films and their AFM coupling to the Fe substrate (grey area in 3): negative magnetic moments in odd-index and positive magnetic moments in even-index Mn layers. 
For a hypothetical L-AFM Mn bulk, the local magnetic moments in adjacent layers would be of the same modulus, say $m_{b}$, but with opposite orientation $\left(\ldots,+m_{b},-m_{b},+m_{b},-m_{b}, \ldots\right)$ [39]. The magnetic moments in the central part of a Mn film, however, are subject to two biasing mechanisms: one originates from the Fe substrate, the other from the Mn surface, as will be discussed now.

Keeping in mind the tendency of Mn towards antiferromagnetism, Mn layers in the vicinity of the Fe substrate are expected to be 'biased' in opposite direction of the Fe magnetization, i.e., layers with even index $l$ should have a smaller moment than in bulk bct $\operatorname{Mn}\left[m_{l}<m_{b}\right]$, whereas the moments of layers with odd index should be increased (in absolute value; $m_{l}<-m_{b}$ ). In other words, the Fe substrate turns $\mathrm{Mn}$ from an in-layer antiferromagnetic into an in-layer ferrimagnetic phase.

On the other hand, the Mn surface layer (layer $n$ of a $n$-ML film) displays an enhanced magnetic moment (compared to the layers underneath), as is found for the Fe surface, too. For films thicker than two layers, the surface moment remains almost constant (in absolute value) at about $3.71 \mu_{\mathrm{B}}$. Introducing a layer-dependent shift $\delta m_{l}$ of the surface-layer magnetic moment (with respect to the Mn bulk), the moments in films with an even number of layers can be written as $m_{l}= \pm m_{b}+\delta m_{l}$, where $+(-)$ is taken for even-index (odd-index) layers and $\delta m_{l}>0$. For a film with an odd number of layers, one has $m_{l}= \pm m_{b}-\delta m_{l}$. To give an illustration: the magnetic profiles at the surface are slightly bent upwards (downwards) for films with an even (odd) numbers of layers (cf the short curved lines in the 11 and 12 ML films in figure 3).

Both biasing effects appear separated in sufficiently thick Mn films (cf the $11 \mathrm{ML}$ and the $12 \mathrm{ML}$ film in figure 3). To be more specific, for films thicker than $6 \mathrm{ML}$, one has $m_{\mathrm{S}}^{\text {even }} \approx-m_{\mathrm{S}}^{\text {odd }}$, i.e., no significant bias at the surface layer $\mathrm{S}$ due to the Fe substrate. For the thinner films, however, one has $-m_{\mathrm{S}}^{\text {even }}>m_{\mathrm{S}}^{\text {odd }}$, which means that the biasing by the Fe also affects the surface magnetic moment $m_{\mathrm{S}}$. This finding is consistent with the evolution of the charge profiles (section 3.1). Because finite-size effects (e.g., quantum-well states) are of minor importance, the profiles can be well superposed by an interface profile, a surface profile, and a Mn-bulk profile, as was theoretically shown for Ni films on $\mathrm{Cu}(001)$ using Green functions [37].

Finally, a few results for the magnetic moments at the surface and the interface are presented. For the uncovered Fe surface (top in figure 3), the surface magnetic moment is enhanced by about $34 \%$ with respect to the bulk magnetic moment $\left(2.99 \mu_{\mathrm{B}}\right.$ versus $\left.2.24 \mu_{\mathrm{B}}\right)$. A similar increase was also found for $\mathrm{Fe}(110)$ [40] (see also [41] for an overview). Upon coverage with $\mathrm{Mn}$, the maximum magnetic moment in the $\mathrm{Fe}$ is shifted from layer 0 to layer -1 , with the increase reduced to about $12 \%$. The reduction of magnetic moment in the top Fe-substrate layer upon Mn coverage has already been found by Spišák and Hafner and has been explained by the competition between antiferromagnetic $\mathrm{Mn}-\mathrm{Fe}$ and ferromagnetic $\mathrm{Fe}-\mathrm{Fe}$ interactions [42]. In layer 1 at the $\mathrm{Mn}-\mathrm{Fe}$ interface, the lowest $\mathrm{Mn}$ magnetic moment is observed for the $1 \mathrm{ML}$ film $\left(-3.78 \mu_{\mathrm{B}}\right)$. With increasing film thickness, the moment of this layer increases to about $-2.52 \mu_{\mathrm{B}}$.

Recently, Martínez et al reported on first-principles calculations for 6 and 7 ML $\mathrm{Mn} / \mathrm{Fe}(001)$ [30]. They find several collinear magnetic configurations in the Mn film, the total-energy differences among which are small. Hence, the authors concluded that various magnetic orderings could coexist at room temperature. We would like to note that the findings of Martínez et al are similar to ours, in particular with respect to the enhanced Mn surface magnetic moment and a tendency to L-AFM coupling within the Mn film. Differences, especially an FM coupling between the Fe substrate and the adjacent Mn layer, could possibly be explained by approximations inherent to the calculations, one of which might be particularly important. 
Martínez et al used a slab geometry (supercell) while in the present investigation appropriate boundary conditions for a semi-infinite system were taken into account.

In the charge profile of the $9 \mathrm{ML}$ film (figure 2), the differences of the spin-resolved valence charges of the d electrons in layers 5, 7, and 8 (layers S-4, S-2, and S-1 in conventional nomenclature) appear reduced with respect to those in the other Mn layers (valence charges of approximately 3.5 electrons and 2.0 electrons as compared to 4.0 electrons and 1.5 electrons, respectively). Hence, the magnetic moments in these layers are reduced in absolute value. A closer look at the data shows that Mn films 7-12 ML thick display this behaviour at layers S-1, S-2, and S-4, too. Although the magnetic profiles presented in figure 3 reveal an at first glance almost regular shape (cf the above modelling), they are rather complicated in detail (cf [30]). This behaviour seems to reflect the location of Mn between an antiferromagnet (Cr) and a ferromagnet $(\mathrm{Fe})$ in the periodic table, making it difficult to pinpoint an unequivocal origin of this finding.

Considering both the charge and magnetic profiles, one can conclude that the film thickness up to which finite-size effects show up is about six layers. This finding implies for firstprinciples calculations that $\mathrm{Mn} / \mathrm{Fe}(001)$ might not be simulated well by a comparably thin slab of Mn layers (i.e., a stack of Mn layers without Fe substrate). In particular, the surface magnetic structures obtained by a slab and a film-substrate calculation might differ. Therefore, surface states, which are obviously sensitive to surface properties, could occur at different energies. This issue will be addressed in the forthcoming sections.

\subsection{Density of states}

The electronic structure of a film is characterized by the layer- and spin-resolved density of states (DOS) $D_{l}^{\tau}(E)$, shown here as an example for the $9 \mathrm{ML}$ film (figure 4). The Mn layers with even index $l$ show most spectral weight below the Fermi energy for majority spin (cf layers S-1, S-3, S-5, and S-7 between about -4 and $-1 \mathrm{eV}$ ), whereas the odd-index Mn layers display significant weight for minority spin in the same energy range. Above the Fermi energy, between about 0 and $+2 \mathrm{eV}$, the odd-index (even-index) layers show significant weight in the majority (minority) DOS. This alternating behaviour is a clear manifestation of the L-AFM structure (as indicated schematically by horizontal arrows on the right in figure 4), as can be understood from the following. Modelling the L-AFM structure by means of a KronigPenney model [43], the electrons experience a potential with alternating height. Thus, the charge density for electrons of one spin orientation is larger in layers with lower potential than in layers with higher potential, i.e., they become more localized in the 'potential wells' than in the 'potential barriers'. For the majority electrons in the $9 \mathrm{ML}$ film, these are the even-index layers. Electrons with the opposite spin orientation are subject to the same potential but shifted by one layer.

The apparent exchange splitting $E_{\mathrm{ex}}$ in $\mathrm{Mn}$, taken as the energy difference between the main spin-resolved spectral weight (at about -2.1 and $+0.9 \mathrm{eV}$; cf the arrow in figure 4(b) at layer $\mathrm{Mn} \mathrm{S-2)}$ is estimated to be $3 \mathrm{eV}$, in reasonable agreement with $2.9 \mathrm{eV}$ obtained from a SPEELS experiment [21]. A comparison of the theoretical surface magnetic moments with these SPEELS data appears-due to the rather complicated magnetic profile in theory and the surface averaging inherent in the experiment—not to be very promising. The theoretical values, at least, do not contradict the experimental finding of $2.9 \mu_{\mathrm{B}}$.

The results for the charge and the magnetic profiles suggest that the Mn films appear to be rather inert, or in other words, that Fe electronic states do not hybridize significantly with Mn states. This is corroborated by the DOS: sharp maxima in the outermost Fe layer 0 (S-9) do not show up in the adjacent Mn layer 1 (S-8), and vice versa. 

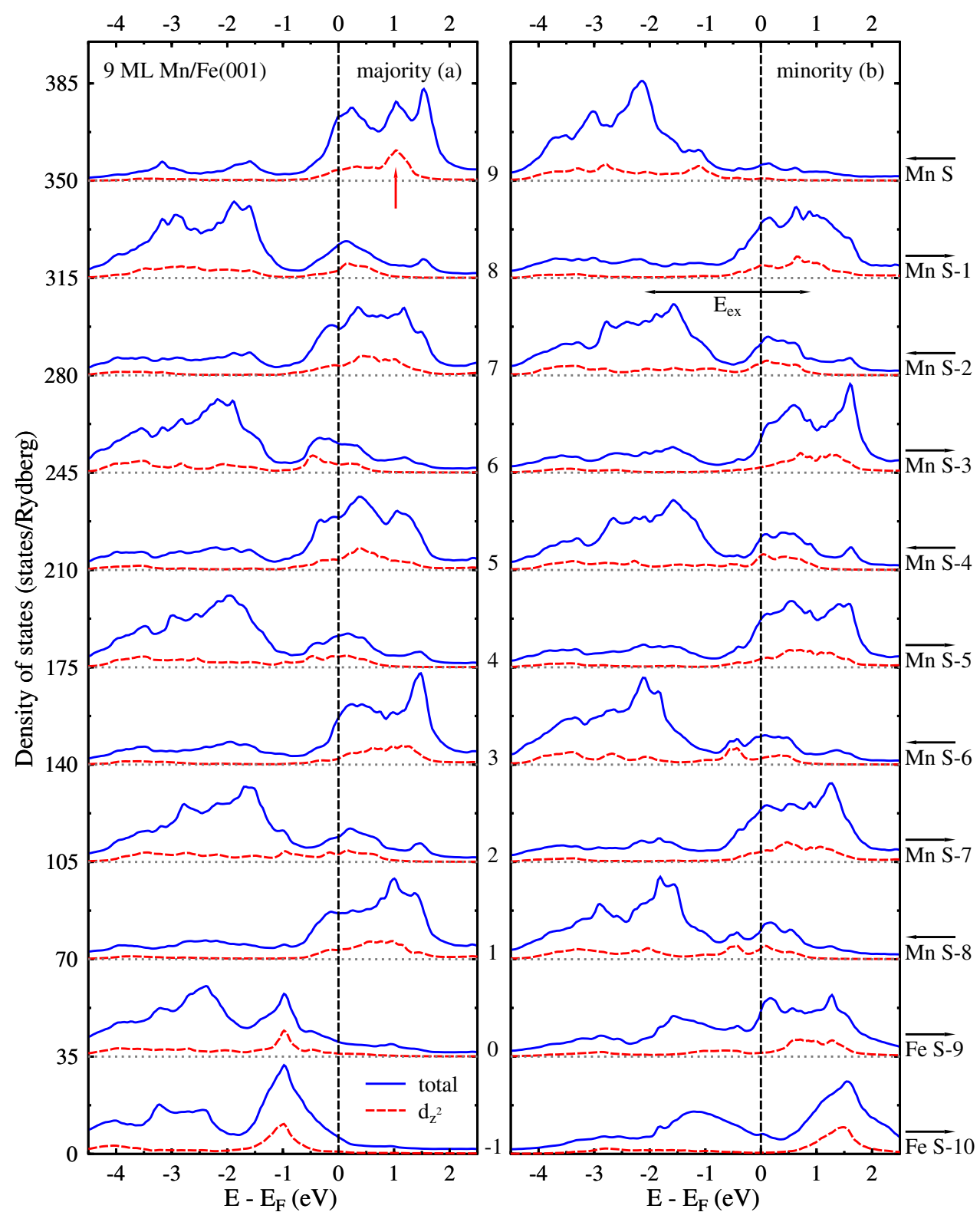

Figure 4. Density of states $D_{l}^{\tau}(E)$ of $9 \mathrm{ML} \mathrm{Mn} / \mathrm{Fe}(001)$, resolved with respect to spin orientation $\tau$ (left, majority (a); right, minority (b)) and layer $l$. The $\mathrm{d}_{z^{2}}$ contribution is shown in addition (dashed). The layer index $l$ is given in numerical form between the panels and in conventional nomenclature on the right (where S stands for 'surface'), with horizontal arrows at the latter indicating schematically the orientations of the magnetic moments. The vertical arrow in layer $\mathrm{Mn} S$ refers to a majority surface feature discussed in the text. The apparent exchange splitting $E_{\mathrm{ex}}$ is displayed in (b) by a horizontal arrow. The offset between adjacent spectra is 35 states Ryd $^{-1}$; the Fermi energy $E_{\mathrm{F}}$ is marked by a dashed vertical line.

As is evident from the charge profiles, especially from figure 2, the main contribution to the DOS stems from d electrons, whereas $\mathrm{s}$ and $\mathrm{p}$ electrons make up a minor part. In view 
of the occurrence of surface states, the DOS was further resolved with respect to $\mathrm{d}_{z^{2}}$ orbitals. In the surface layer 9 (Mn S), a $\mathrm{d}_{z^{2}}$ maximum shows up around $+1 \mathrm{eV}$ in the majority DOS (vertical arrow in figure 4(a)). This comparably broad feature can be related to the maximum at $+0.8 \mathrm{eV}$ which Yamada et al found in STS $\mathrm{d} I / \mathrm{d} V$-spectra [31, 32]. (Note that this maximum would appear in the minority DOS if the magnetic moment of the Mn surface layer were taken as a reference, as was done in [32].) In STS only a small part of the $2 \mathrm{BZ}$ contributes to the tunnel current, whereas the DOS averages over the entire Brillouin zone (cf (2)). Therefore, an analysis of the electronic structure at the $2 \mathrm{BZ}$ centre, which can be expected to contribute essentially to the STS signal, is needed.

\subsection{Spectral density at $\bar{\Gamma}$}

The relevant point group at $\bar{\Gamma}\left(\boldsymbol{k}_{\|}=0\right)$ is $\mathrm{C}_{4 \mathrm{v}}$ (in Schoenflies notation) which possesses the representations $\Delta_{1}, \Delta_{1^{\prime}}, \Delta_{2}, \Delta_{2^{\prime}}$, and $\Delta_{5}$ [44]. Due to exchange splitting, these become spin dependent, leading to spin-resolved representations $\Delta_{i}^{\tau}, \tau=\uparrow, \downarrow$. The spin-quantization axis is chosen as that of the magnetization direction of the Fe substrate. Of specific interest are the representations $\Delta_{1}^{\tau}$ which comprise $\mathrm{p}_{z}$ and $\mathrm{d}_{z^{2}}$ orbitals. The latter were related in [32] to the experimentally observed maxima in STM $\mathrm{d} I / \mathrm{d} V$-spectra because of their spatial orientation towards the vacuum. The other representations comprise $\mathrm{d}_{x^{2}-y^{2}}\left(\Delta_{2}^{\tau}\right), \mathrm{d}_{x y}\left(\Delta_{2^{\prime}}^{\tau}\right)$, and $\mathrm{d}_{z x}$ as well as $\mathrm{d}_{z y}\left(\Delta_{5}^{\tau}\right) \mathrm{d}$ orbitals.

The spectral density of a $6 \mathrm{ML}$ film is shown in figure 5. As also found for the $9 \mathrm{ML}$ film (figure 4), the Mn layers exhibit an alternating behaviour in the main spectral weight, a consequence of the L-AFM structure.

The hybridization between the $\mathrm{Fe}$ and the Mn electronic states is rather small, as is evident from the spectral densities of layers 0 (Fe S-6) and 7 (Mn S-5). For example, Fe states do not penetrate the Mn film, i.e., they produce no significant maxima in layer 7. Vice versa, Mn states show up only as minor peaks in layer 6 . The small magnetic coupling between Fe and $\mathrm{Mn}$ is consistent with calculations for a Heisenberg model performed by Schlickum [45].

Focusing now on the surface layers, one does not find maxima which display the typical behaviour of a surface state in a nonmagnetic or a ferromagnetic system, that is, maximum spectral weight in the outermost layer which decays exponentially towards the bulk. The most promising candidates for majority surface states show up at energies of $-2.10 \mathrm{eV}\left(\Delta_{1}^{\uparrow}\right)$, $-2.04 \mathrm{eV}\left(\Delta_{2,2^{\prime}}^{\uparrow}\right),-1.63 \mathrm{eV}\left(\Delta_{5}^{\uparrow}\right)$, and $0.80 \mathrm{eV}\left(\Delta_{1}^{\uparrow}\right)$, all marked by vertical lines in figure 5 . For minority spin, candidates appear at $-3.60 \mathrm{eV}\left(\Delta_{1}^{\downarrow}\right),-1.16 \mathrm{eV}\left(\Delta_{5}^{\downarrow}\right),-0.59 \mathrm{eV}\left(\Delta_{2,2^{\prime}}^{\downarrow}\right)$, $+0.78 \mathrm{eV}\left(\Delta_{1}^{\downarrow}\right)$, and $+1.88 \mathrm{eV}\left(\Delta_{2,2^{\prime}}^{\downarrow}\right)$. The minority state at $+0.78 \mathrm{eV}$ shows an unusual behaviour (compared to the others) since it is located within the three outermost layers (with indices $l=4,5$, and 6). To elucidate the surface electronic in more detail we compare the results for the $6 \mathrm{ML}$ film with that of a $12 \mathrm{ML}$ film (figure 6).

Upon increasing the number of layers in a film, one obtains more maxima in the layerresolved spectral density for each bulk band, in accordance with tight-binding theory. This effect also shows up in $\mathrm{Mn} / \mathrm{Fe}(001)$ when doubling the number of Mn layers from six (figure 5) to 12 (figure 6). If the film is sufficiently thick, surface states should not be affected, except to a possible small shift in energy (which is due to the slightly different reflection at the substrateside layers). Comparing the spectral densities for the outermost Mn layers S and S-1, one finds for each surface-state candidate of the $6 \mathrm{ML}$ film a counterpart in the $12 \mathrm{ML}$. The maximum deviation is less than $0.05 \mathrm{eV}$. An exception seems to be the $\Delta_{1}^{\downarrow}$ state at $+0.78 \mathrm{eV}$ (6 ML film) or $+0.89 \mathrm{eV}$ (12 ML film): The energy shift is rather large compared to those of the other surface states. 

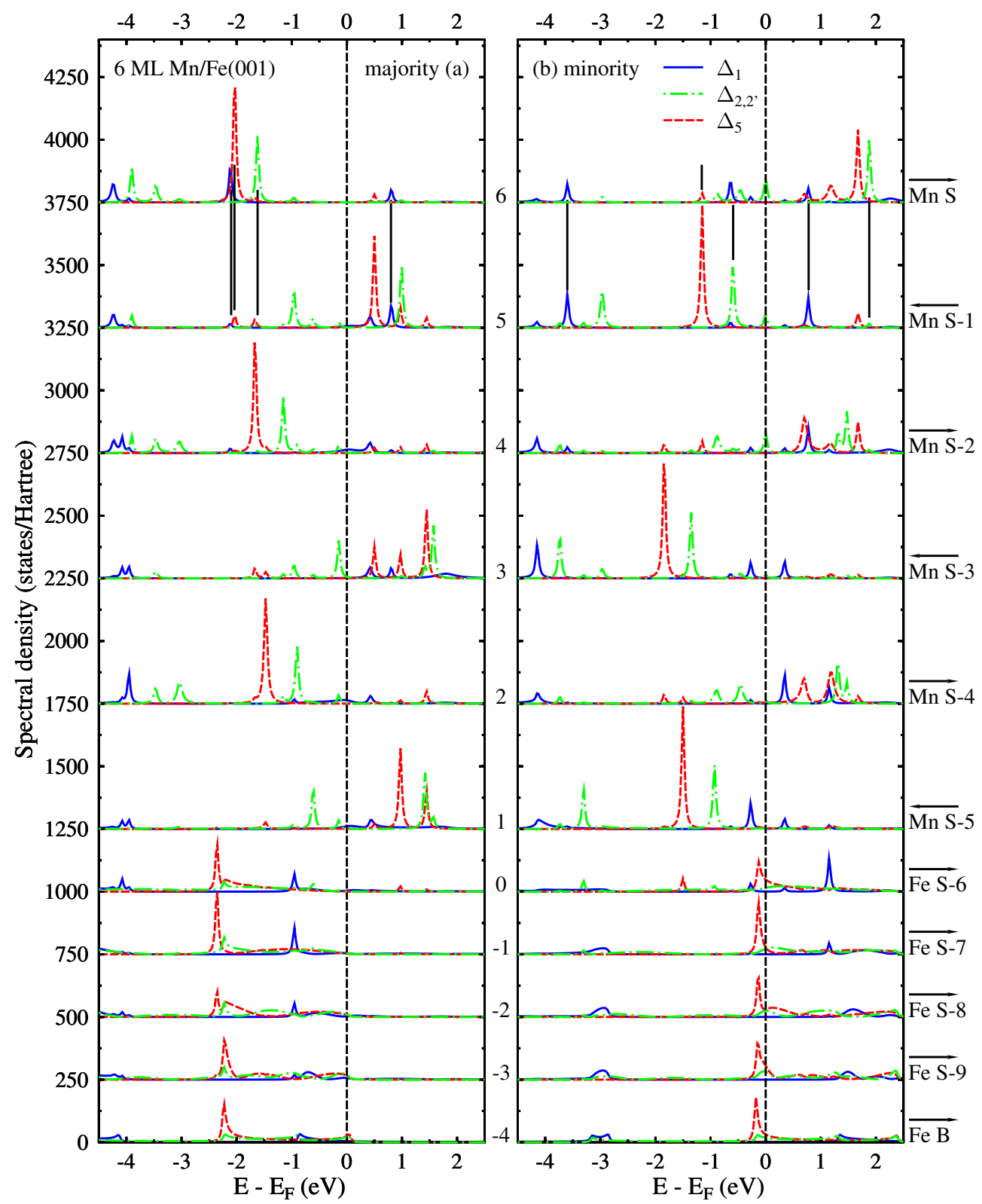

Figure 5. Spectral density $N_{l}^{\tau}\left(E, \boldsymbol{k}_{\|}\right)$of $6 \mathrm{ML} \mathrm{Mn} / \mathrm{Fe}(001)$ at $\bar{\Gamma}$ (i.e., $\boldsymbol{k}_{\|}=0$ ). The spectral density is resolved with respect to spin orientation $\tau$ (left, majority (a); right, minority (b)), single-group representation ( $\Delta_{1}$ solid, $\Delta_{2,2^{\prime}}$ dashed-dotted, and $\Delta_{5}$ dashed), and layer $l$ (as in figure 4 ; B stands for 'bulk'). Surface states are marked by vertical lines at layers 5 (Mn S-1) and 6 (Mn S). The offset between adjacent spectra is 250 states/Hartree in Fe and 500 states/Hartree in Mn. The Fermi energy $E_{\mathrm{F}}$ is marked by a dashed vertical line.

A further test on a surface state is that it lies energetically in a bandgap of the bulk states. Taking the potentials of the central Mn layers of the film, the bulk bands of this hypothetical 'cut and paste' L-AFM structure were computed (top panels in figure 6). If the magnetic moments 

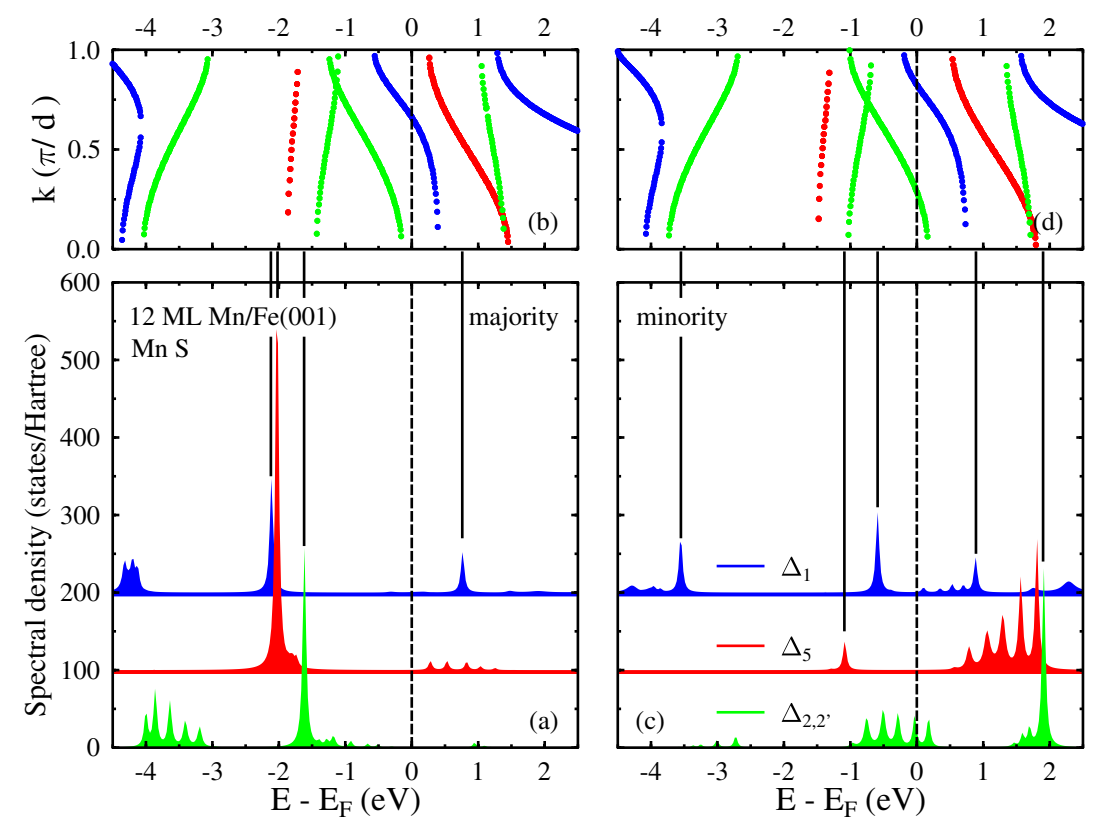

Figure 6. Surface states on $12 \mathrm{ML} \mathrm{Mn/Fe}(001)$ at $\bar{\Gamma}$. Bottom row: the spectral densities $N_{l}^{\tau}\left(E, \boldsymbol{k}_{\|}\right)$ for the surface layer 'Mn S' are shown in (a) and (c). The offset between adjacent spectra is 100 states/Hartree. Surface states are marked by vertical solid lines, indications as in figure 5. Top row: the band structure of in-layer AFM Mn bulk is displayed in (b) and (d), with the same coding as for the spectral densities. The interlayer distance is denoted $d$.

in the two constituting layers were identical, all bands would be twofold degenerate [39]. However, due to the biasing mechanism discussed in section 3.2, this degeneracy is lifted (with local magnetic moments of 2.72 and $-1.93 \mu_{\mathrm{B}}$; figure 3 ), and the bands can be classified into minority and majority bands. The former are shifted by about $0.3 \mathrm{eV}$ to larger energies with respect to the latter (top panels in figure 6).

Analysing the energy positions of the states under consideration with respect to the bulk bands, one finds that all are located within symmetry bandgaps. Regarding their energetic distance from the band edges of bulk bands with the same symmetry, one can classify the surface states with respect to their origin as Tamm or Shockley states [46]. Tamm states appear due to truncation of the solid and, hence, are split off the associated bulk band, whereas Shockley states appear due to formation of bonding and anti-bonding states when assembling a solid from atoms. (We are aware that this historical classification appears nowadays-in the times of first-principles calculations-somewhat artificial.) As an example, the $\Delta_{1}^{\downarrow}$ state at $0.89 \mathrm{eV}$ is split off the $\Delta_{1}^{\downarrow}$ band which crosses the Fermi level (with the upper band edge at $+0.79 \mathrm{eV}$ ), and, hence, can be regarded as a Tamm surface state. The maximum in the $\mathrm{d} I / \mathrm{d} V$-spectra at $+0.8 \mathrm{eV}$ reported by Yamada [32] might be explained by this surface state. Considering the distribution of its spectral weight over the few outermost layers (at least three layers; see also [39]) it is evident that for an accurate numerical description one needs both a sufficiently thick film and correct boundary conditions.

Surface-state positions are related to the surface magnetic moment, as was theoretical investigated by Halilov et al by means of a model calculation [39]. Since the surface magnetic moment is much larger than in the centre of the Mn film, two (Tamm) surface states could 
appear: one below the lower partner and the other above the upper partner of a bulk-band pair (cf figure 1 in [39]). For example, a pair of $\Delta_{2,2^{\prime}}^{\uparrow}$ bands shows up between -1.5 and $-1.0 \mathrm{eV}$ (lower partner; green in figure $6(\mathrm{~b})$ ) and between +1.3 and $+1.7 \mathrm{eV}$ (upper partner; figure 6(d)). The associated surface states have energies of $-1.62 \mathrm{eV}$ (figure 6(a)), i.e., below the lower partner band, and $+1.90 \mathrm{eV}$ (figure 6(c)), above the upper partner band. Hence, the present first-principles calculation nicely confirms the model calculation.

\section{Concluding remarks}

The present theoretical work focuses mainly on thicker Mn films, in which the in-layer AFM structure was also found in experiments. Open problems to be tackled in a future publication comprise the magnetic anisotropy of the Mn atoms, in particular in view of non-collinear magnetic phases. Within this respect, a comparison with $\mathrm{Cr} / \mathrm{Fe}(001)$ would be very interesting.

Further experimental investigations on $\mathrm{Mn} / \mathrm{Fe}(001)$ which are highly desirable could comprise spin-resolved scanning tunnelling microscopy and spectroscopy $[47,48]$ as well as photoelectron spectroscopy [49]. The former could directly prove the L-AFM structure of the Mn films, detect surface states, and look for signatures of the magnetic structure in the tunnel magnetoresistance [50]. For photoemission one can-besides spin-resolved experimentsthink about magnetic linear dichroism from the valence bands [51,52]. Considering trends in both change of sign and magnitude of the intensity asymmetry, the magnetic structure could be deduced.

\section{Acknowledgments}

We are grateful to U Schlickum, W Wulfhekel, and J Kirschner for valuable discussions.

\section{References}

[1] Frenken J W M, Horn K, Le Lay G and Woodruff D P (ed) 2003 Growth \& Evolution of Ultrathin Films: Surface \& Interface Geometric \& Electronic Structure (Amsterdam: Elsevier)

[2] Bland J A C and Heinrich B 1994 Ultrathin Magnetic Structures (Berlin: Springer)

[3] Hartmann U (ed) 1999 Magnetic Multilayers and Giant Magnetoresistance. Fundamentals and Industrial Applications (Springer Series in Surface Sciences vol 37) (Berlin: Springer)

[4] Blügel S, Drittler B, Zeller R and Dederichs P H 1989 Appl. Phys. A 49547

[5] Mirbt S, Eriksson O, Johansson B and Skriver H L 1995 Phys. Rev. B 5215070

[6] Wu R and Freeman A J 1996 J. Magn. Magn. Mater. 16189

[7] Roth C, Kleeman T, Hillebrecht F U and Kisker E 1995 Phys. Rev. B 52 R15691

[8] Dürr H A, van der Laan G, Spanka D, Hillebrecht F U and Brookes N B 1997 Phys. Rev. B 568156

[9] Wu R and Freeman A J 1995 Phys. Rev. B 5117131

[10] Asada T, Bihlmayer G, Handschuh S, Heinze S, Kurz P and Blügel S 1999 J. Phys.: Condens. Matter 119347

[11] Zenia H, Bouarab S, Ferrer J and Demengeat C 2004 Surf. Sci. 56412

[12] Stoeffler D and Gautier F 1993 J. Magn. Magn. Mater. 121259

[13] Purcell S T, Johnson M T, McGee N W E, Coehoorn R and Hoving W 1992 Phys. Rev. B 4513064

[14] Oguchi T and Freeman A J 1984 J. Magn. Magn. Mater. 46 L1

[15] Krüger P, Elmouhssine O, Demangeat C and Parlebas J C 1996 Phys. Rev. B 546393

[16] Vega A, Parlebas J C and Demengeat C 2003 Handbook of magnetic Materials vol 15, ed K H J Buschow (Amsterdam: Elsevier) p 199

[17] Andrieu S, Finazzi M, Yubero F, Fischer H M, Arcade P, Chevrier F, Hennet L, Hricovini K, Krill G and Piecuch M 1997 Europhys. Lett. 38459

[18] Rader O, Gudat W, Schmitz D, Carbone C and Eberhardt W 1997 Phys. Rev. B 565053

[19] Dresselhaus J, Spanke D, Hillebrecht F U, Kisker E, van der Laan G, Goedkoop J B and Brookes N B 1997 Phys. Rev. B 565461 
[20] Igel T, Pfandzelter R and Winter H 1998 Phys. Rev. B 582430

[21] Walker T G and Hopster H 1993 Phys. Rev. B 483563

[22] Tulchinsky D A, Unguris J and Celotta R J 2000 J. Magn. Magn. Mater. 21291

[23] Bischoff M M J 2002 Chemical identification in scanning tunneling microscopy by detection of element-specific surface states PhD Thesis Katholieke Universiteit Nijmegen

[24] Andrieu S, Finazzi M, Bauer P, Fischer H, Lefevre P, Traverse A, Hricovini K, Krill G and Piecuch M 1998 Phys. Rev. B 571985

[25] Andrieu S, Foy E, Fischer H, Almot M and Piecuch M 1998 Phys. Rev. B 588210

[26] Dreyssé H and Demangeat C 1997 Surf. Sci. Rep. 2865

[27] Andrieu S, Finazzi M, Yubero F, Fischer H, Arcade P, Chevrier F, Hricovini K, Krill G and Piecuch M 1997 J. Magn. Magn. Mater. 165191

[28] Bouarab S, Nait-Laziz H, Khan M A, Demengeat C, Dreyseé H and Benakki M 1995 Phys. Rev. B 5210127

[29] Schlickum U, Janke-Gilman N, Wulfhekel W and Kirschner J 2004 Phys. Rev. Lett. 92107203

[30] Martínez E, Vega A, Robles R and Vázquez des Parga A L 2005 Phys. Lett. A 337469

[31] Yamada T K, Bischoff M M J, Mizoguchi T and van Kempen H 2002 Surf. Sci. 516179

[32] Yamada T K, Bischoff M M J, Heijnen G M M, Mizoguchi T and van Kempen H 2003 Phys. Rev. Lett. 90 056803

[33] Kresse G and Furthmüller J 1996 Phys. Rev. B 5411169

[34] Perdew J P and Wang Y 1992 Phys. Rev. B 4513244

[35] Ernst A, Lüders M, Temmerman W M, Szotek Z and van der Laan G 2000 J. Phys.: Condens. Matter 125599

[36] Weinberger P 1990 Electron Scattering Theory of Ordered and Disordered Matter (Oxford: Clarendon)

[37] Henk J, Niklasson A M N and Johansson B 1999 Phys. Rev. B 599332

[38] Halilov S V, Henk J, Scheunemann T and Feder R 1995 Phys. Rev. B 5214235

[39] Halilov S V, Henk J, Scheunemann T and Feder R 1995 Surf. Sci. 343148

[40] Tamura E, Feder R, Waller G and Gradmann U 1990 Phys. Status Solidi b 157627

[41] Gradmann U 1991 J. Magn. Magn. Mater. 100481

[42] Spišák D and Hafner J 1997 Phys. Rev. B 558304

[43] Merzbacher E 1970 Quantum Mechanics 2nd edn (New York: Wiley)

[44] Inui T, Tanabe Y and Onodera Y 1990 Group Theory and its Applications in Physics (Springer Series in Solid State Sciences vol 78) (Berlin: Springer)

[45] Schlickum U 2004 Private communication

[46] Davison S G and Stȩślicka M 1992 Basic Theory of Surface States (Oxford: Clarendon)

[47] Bode M 2003 Rep. Prog. Phys. 66523

[48] Wulfhekel W and Kirschner J 1999 Appl. Phys. Lett. 751944

[49] Hüfner S 2003 Photoelectron Spectroscopy 3rd edn (Berlin: Springer)

[50] Bode M, Getzlaff M and Wiesendanger R 1998 Phys. Rev. Lett. 814256

[51] Henk J, Halilov S V, Scheunemann T and Feder R 1994 Phys. Rev. B 508130

[52] Bansmann J, Lu L, Getzlaff M, Fluchtmann M, Braun J and Meiwes-Broer K H 1998 J. Magn. Magn. Mater. 8594 